\title{
Hydrogen Storage Behaviors of Carbon Nanotubes/Metal-organic Frameworks-5 Hybrid Composites
}

\author{
Soo-Jin Park^ and Seul-Yi Lee \\ Department of Chemistry, Inha University, 253 Nam-gu, Incheon 402-751, South Korea \\ •e-mail: sjpark@inha.ac.kr \\ (Received February 9, 2009; Accepted March 13, 2009)
}

\begin{abstract}
In this work, the hydrogen storage behaviors of carbon nanotubes (CNTs)/metal-organic frameworks-5 (MOF-5) hybrid composites (CNTs/MOF-5) were studied. Hydrothermal synthesis of MOF-5 was conducted by conventional convection heating using 1-methyl-2-pyrrolidone (NMP) as a solvent. Morphological characteristics and average size of the CNTs/MOF5 were also obtained using a scanning electron microscopy (SEM). The pore structure and specific surface area of the CNTs/ MOF-5 were analyzed by $\mathrm{N}_{2} / 77 \mathrm{~K}$ adsorption isotherms. The capacity of hydrogen storage of the CNTs/MOF-5 was investigated at $298 \mathrm{~K} / 100$ bar. As a result, the CNTs/MOF-5 had crystalline structures which were formed by hybrid synthesis process. It was noted that the $\mathrm{CNTs} / \mathrm{MOF}-5$ can be potentially encouraging materials for hydrogen adsorption and storage applications at room temperature.
\end{abstract}

Keywords : Carbon nanotubes, Metal-organic frameworks, Hydrogen storage, XRD, SEM

\section{Introduction}

Hydrogen have considered as a clean energy source due to its almost-zero pollutant emission during combustion. Many efforts are conducted worldwide to find out efficient and cost-effective hydrogen storage materials for mobile applications. An extensive storage methods have been investigated including liquid hydrogen, metal hydrides, electrosorption, high-pressure hydrogen, and physisorption in porous materials $[1,2]$. However, no method as yet has a clear technical and commercial future. Storing molecular hydrogen in porous media is one of the promising way for hydrogen storage. From among these, the physisorption of hydrogen remains an attractive alternative due to its reversible storage reaction and safety [3]. Many researchers have been investigated for physisorption with carbon nanotubes (CNTs), activated carbon, zeolites, and metalorganic frameworks (MOFs) [4-6]. The interest in these materials lies in the fact that they can be optimized for hydrogen storage through various physical and chemical treatments.

Carbon nanotubes (CNTs) are known to offer many advantages such as low mass density and high surface area like other typical carbon materials. In particular, the adsorption of hydrogen in single-walled carbon nanotubes (SWNTs) has also been the object of much controversy. The narrow pore size distribution of SWNTs makes them attractive candidates as adsorbents for hydrogen. Their cylindrical structure would be increases the adsorption potential on the tube core leading to capillary forces and to enhanced storage capability $[7,8]$.

MOFs have the potentials to exhibit properties such as chirality, framework interpenetration, or framework flexibility[9]. Recently, a new class of porous MOFs have attracted much attention due to their potential applications in heterogeneous catalyst, separations, sensors, electronics, and gas storage $[10,11]$. Porous MOFs have also permanent nanoscaled cavities and open channels, to which small molecules can give access with inherent conditions. Their extremely high surface area and accessibility to design and fine-tunable pore structures are also appropriate to optimize for various purposes. In this repect, MOFs have been considered strong candidates for hydrogen storage. Therefore, we employed MOF-5 that is the most stable, widely available, and remarkable porous in comparison with other MOF series. MOF-5 naturally became our initial target for the CNTs/ MOF-5 hybrid composites for hydrogen storage.

In this work, we used multi-walled carbon nanotubes (MWNTs) and single-walled carbon nanotubes (SWNTs) as carbon materials, respectively, and MOF-5 as porous receptors. The objectives of the work are to study not only the hydrogen storage capacity of MOF-5 but also the hydrogen storage behaviors in association with the CNTs (i.e. MWNTs and SWNTs)/MOF-5 hybrid composites by synergetic effect. 


\section{Experimental}

\subsection{Materials and Sample Preparation}

MWNT crude materials (diameter: $10-25 \mathrm{~nm}$ and length: 10-50 $\mu \mathrm{m}$ ) and SWNT crude materials (diameter:1.0-1.2 nm and length : 10-30 $\mu \mathrm{m}$ ) purchased from Nano Solution Co. (Korea). For the purpose of surface treatment, MWNTs and SWNTs were chemically mixed with $5 \mathrm{M}$ nitric acid for $5 \mathrm{~h}$ at room temperature by liquid-phase oxidation. This sample was then filtered, washed, and then dried in a vacuum oven for $24 \mathrm{~h}$ at $120^{\circ} \mathrm{C}$.

The MOF-5 was hydrothermally synthesized using a solution of 1-methyl-2-pyrrolidone (NMP, $\left.\mathrm{C}_{5} \mathrm{H}_{9} \mathrm{NO}, \mathrm{TCI}\right)$ as a solvent. Zinc nitrate $\left(\mathrm{Zn}\left(\mathrm{NO}_{3}\right)_{2} \cdot 6 \mathrm{H}_{2} \mathrm{O}\right.$, Aldrich, 98\%, $\left.2.78 \mathrm{~g}\right)$ and terephthalic acid $\left(\mathrm{C}_{6} \mathrm{H}_{4}(\mathrm{COOH})_{2}\right.$, Aldrich, $\left.98 \%, 0.55 \mathrm{~g}\right)$ were dissolved in $90 \mathrm{ml}$ NMP. The reaction mixture was transferred to Teflon-lined steel autoclave and heated at a heating rate of $1^{\circ} \mathrm{C} / \mathrm{min}$ with temperatures rising to $105^{\circ} \mathrm{C}$ and then the temperature was held $24 \mathrm{~h}$. The white and slightly pale yellow powdered product was synthesized, which washed with dried NMP for removal of the residual reactant and then was solvent-exchanged with chloroform. The solid crystals finally vacuum-dried at $100^{\circ} \mathrm{C}$ for $24 \mathrm{~h}$.

Both acid-treated MWNTs and SWNTs were dispersed in NMP by sonication. Each solution mixed with acid-treated MWNTs and SWNTs was put into the starting mixture for MOF-5. The synthesized MOFs sample ware labeled as MOF-5, the acid-treated MWNTs/MOF-5 hybrid composites were M-MOF-5, and the acid-treated SWNTs/MOF-5 hybrid composites were S-MOF-5.

\subsection{Characterization and Hydrogen Storage Capacity}

X-ray diffraction (XRD) patterns were collected on a Rigaku Model D/MAX diffractometer at $40 \mathrm{kV}$ and $20 \mathrm{~mA}$

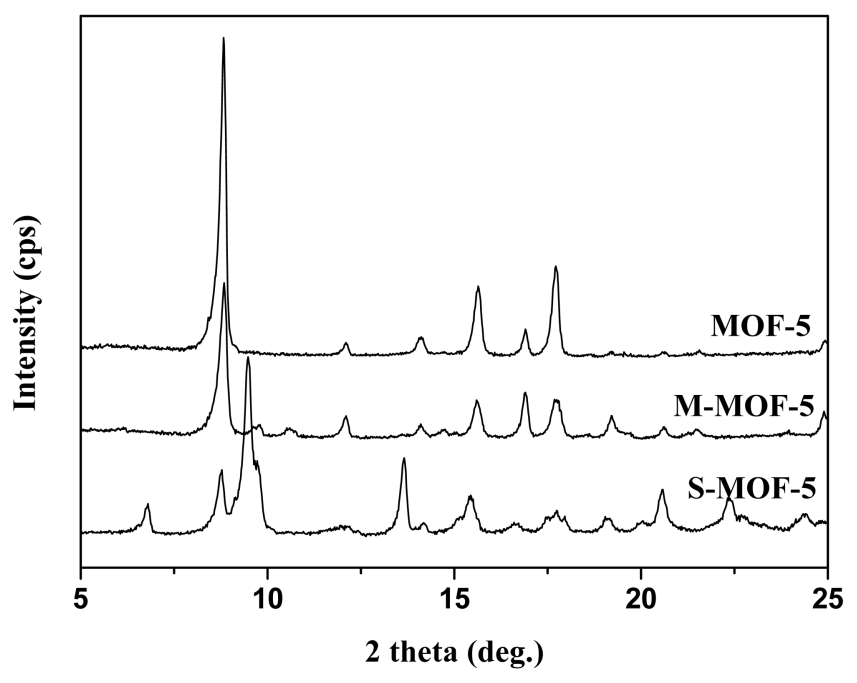

Fig. 1. XRD patterns of MOF-5, M-MOF-5, and S-MOF-5. for $\operatorname{CuK}(\lambda=0.1543 \mathrm{~nm})$ radiation. The morphology and structure of the sample were studied with scanning electron microscope (SEM) images using a Hitachi Co., Ltd.'s Model S-4200. The nitrogen isotherms were measured at $77 \mathrm{~K}$ using a Micromeritics' Model TriStar 3000. The samples were out-gassed at $573 \mathrm{~K}$ for $6 \mathrm{~h}$ to obtain a residual pressure of less than $10^{-3}$ torr.

The hydrogen uptake experiment was conducted under an ambient condition of $298 \mathrm{~K}$ and both moderate and low pressure. In each experiment, about $0.5 \mathrm{~g}$ of sample was loaded into a stainless chamber. Preparatory to the measurement, the system was evacuated for $12 \mathrm{~h}$ while heating the sample chamber to $473 \mathrm{~K}$. After the chamber was cooled to room temperature, hydrogen was introduced up to a pressure of 100 bar was attained.
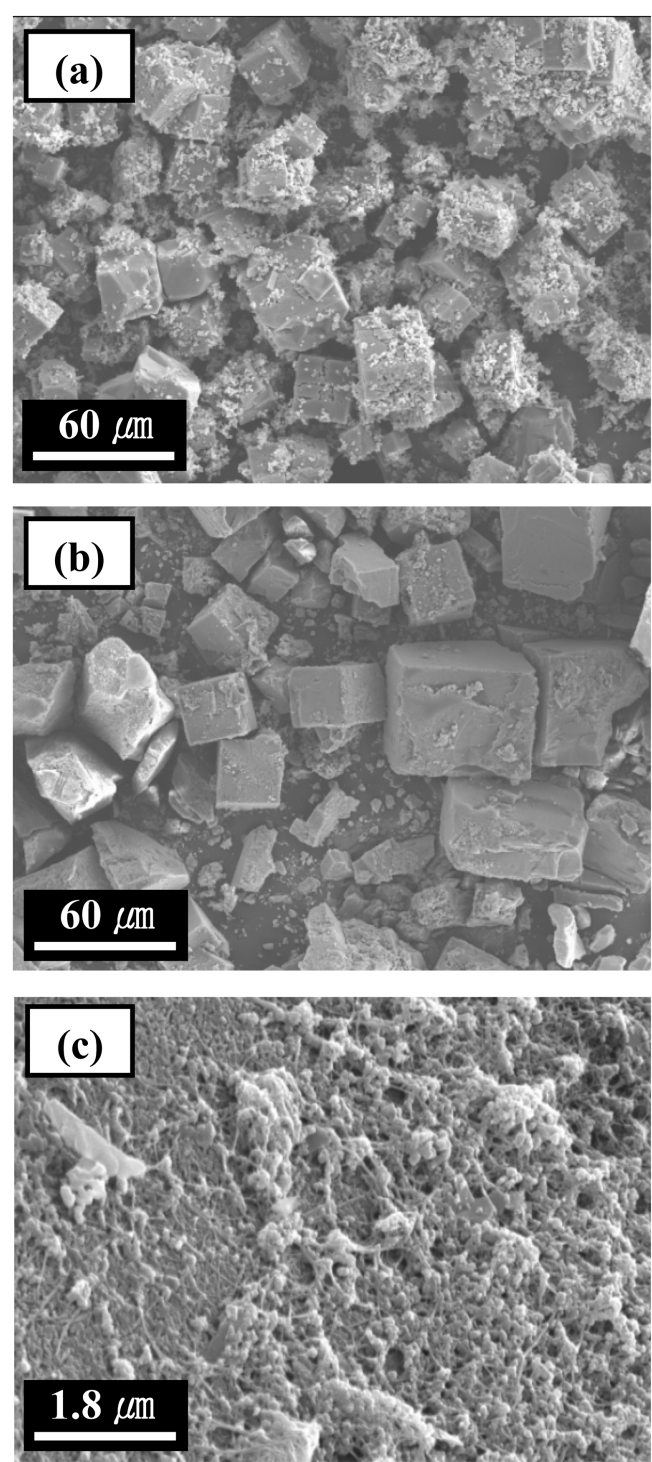

Fig. 2. SEM images of (a) MOF-5, (b) S-MOF-5, and (c) the magnification of S-MOF-5 surfaces. 
Table 1. $\mathrm{N}_{2} / 77 \mathrm{~K}$ textural properties of MOF-5, M-MOF-5, and S-MOF-5

\begin{tabular}{ccccccc}
\hline Specimens & ${ }^{\mathrm{a}} \mathrm{S}_{\mathrm{BET}}\left(\mathrm{m}^{2} / \mathrm{g}\right)$ & ${ }^{\mathrm{b}} \mathrm{V}_{\text {Total }}\left(\mathrm{cm}^{3} / \mathrm{g}\right)$ & ${ }^{\mathrm{c}} \mathrm{V}_{\text {Micro }}\left(\mathrm{cm}^{3} / \mathrm{g}\right)$ & ${ }^{\mathrm{d}} \mathrm{V}_{\text {Meso }}\left(\mathrm{cm}^{3} / \mathrm{g}\right)$ & ${ }^{\mathrm{e}} \mathrm{D}(\mathrm{nm})$ & ${ }^{\mathrm{f}} \mathrm{F}_{\text {Micro }}(\%)$ \\
\hline MOF-5 & 812 & 0.645 & 0.211 & 0.434 & 3.5 & 32.7 \\
M-MOF-5 & 343 & 0.442 & 0.047 & 0.395 & 5.1 & 10.6 \\
S-MOF-5 & 720 & 0.463 & 0.194 & 0.269 & 2.5 & 41.9 \\
\hline
\end{tabular}

${ }^{a} \mathrm{~S}_{\mathrm{BET}}$ : Specific surface area computed using BET equation at a relative pressure of 0.29 .

${ }^{\mathrm{b}} \mathrm{V}_{\text {Total }}$ : Total pore volume is estimated at a relative pressure $\mathrm{P} / \mathrm{P}_{0}=0.99$.

${ }^{\mathrm{c}} \mathrm{V}_{\text {Micro }}$ : Micropore volume determined from the subtraction of mesopore volume from total pore volume.

${ }^{\mathrm{d}} \mathrm{V}_{\text {Meso }}$ : Mesopore volume determined from the BJH method.

${ }^{\mathrm{e}} \mathrm{D}$ : Average pore size determined from BJH desorption method.

${ }^{\mathrm{f}} \mathrm{F}_{\text {Micro }}$ : Fraction of micropore volume $=($ micropore volume $/$ total pore volume $) * 100$

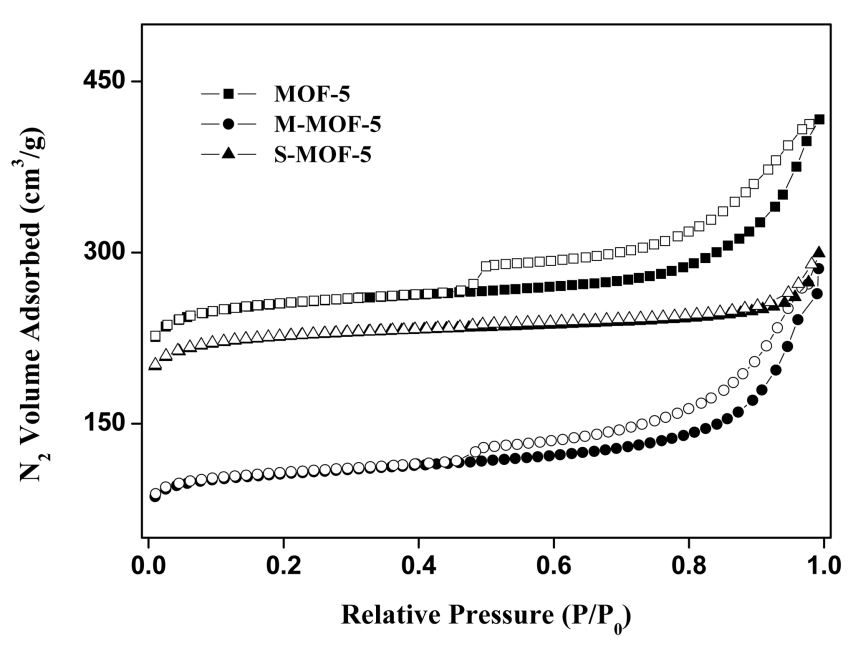

Fig. 3. Nitrogen physisorption isotherms of MOF-5, M-MOF-5, and S-MOF-5.

\section{Results and Discussion}

\subsection{Characterization}

Fig. 1 illustrates XRD spectra of MOF-5, M-MOF-5, and S-MOF-5. The extremely high intensities of the diffraction peaks indicate the good crystallinity of all samples. The strongest peak at $2 \theta=8.8^{\circ}$ and the peaks between $2 \theta=12^{\circ}$ and $14^{\circ}$ in the spectra correspond with the already published XRD patterns [12-14]. Furthermore, the MWNTs and SWNTs themselves cannot be found in the XRD pattern of M-MOF-5 and S-MOF-5, respectively, because the typical reflection plane of graphite structure at $2 \theta=26^{\circ}$ is missing.

More structural details for samples are investigated by SEM observations. As shown in Fig. 2, it is found that all samples have cubically shaped crystals in the range 30-100 mm. Fig. 2 (a) presents that the small- and large-sized crystalline MOF-5 are coexistence with together. The smallsized MOF-5 is scattered on the surfaces of the large-sized MOF-5 and all around. In Fig. 2 (b), it is also confirmed that S-MOF-5 has been grown successfully during the impregnation of SWNTs, which is suggested the presence of
SWNTs on MOF-5 had no influence on textural characteristics. Fig. 2 (c) presented that the surface of SMOF-5 is composed of MOF-5 and SWNT bundles.

The textural properties of MOF-5, M-MOF-5, and SMOF-5 are analyzed based on the $\mathrm{N}_{2}$ physisorption isotherms at $77 \mathrm{~K}$, and are given in Table 1. And, Fig. 3 shows typical isotherms of the $\mathrm{N}_{2}$ adsorption/desorption properties of MOF-5, as previously reported in other studies $[13,15]$. MOF-5 shows high specific surface area and total pore volume in comparison with M-MOF-5 and S-MOF-5, which have specific surface area of $812 \mathrm{~m}^{2} / \mathrm{g}$ and total pore volume of $0.645 \mathrm{~cm}^{3} / \mathrm{g}$. As expected, it can be seen that the adsorption volume is diminished with each impregnation of MWNTs and SWNTs, due to the pore blocking effect and aggregation of MWNTs and SWNTs themselves. Meanwhile, it is interesting to note that the micropore fraction is the highest in the S-MOF-5, which is probably attributable to improve the hydrogen storage capacity.

\subsection{Hydrogen Storage Capacity}

Fig. 4 shows the hydrogen storage capacity of MOF-5, MMOF-5, and S-MOF-5 at $298 \mathrm{~K} / 100$ bar. It is observed that S-MOF-5 shows the highest hydrogen storage capacity than the other samples. Because CNT provides abundant additional interface sites with significant catalytic activity by the high-aspect-ratio characteristics, resulting from the and bonds in association with carbon of graphitic sheets [16]. So hydrogen molecules are adsorbed and diffused into the pores of CNTs as well as the intergrated other porous materials due to the physisorption of hydrogen molecules by weak van der Waals forces. It is indicated that the CNTs/MOF-5 hybrid composites have largely improving the hydrogen storage capacity by synergetic effect, meanwhile the hydrogen storage capacity of M-MOF-5 is a little decreased. It is certain that the presence of CNTs can definitely encourage the hydrogen storage capacity of the CNTs/MOF-5 hybrid composites.

Normally, gas adsorption depends strongly on the specific surface area of support materials because gas adsorption occurs mainly in the micropores of these materials. As 


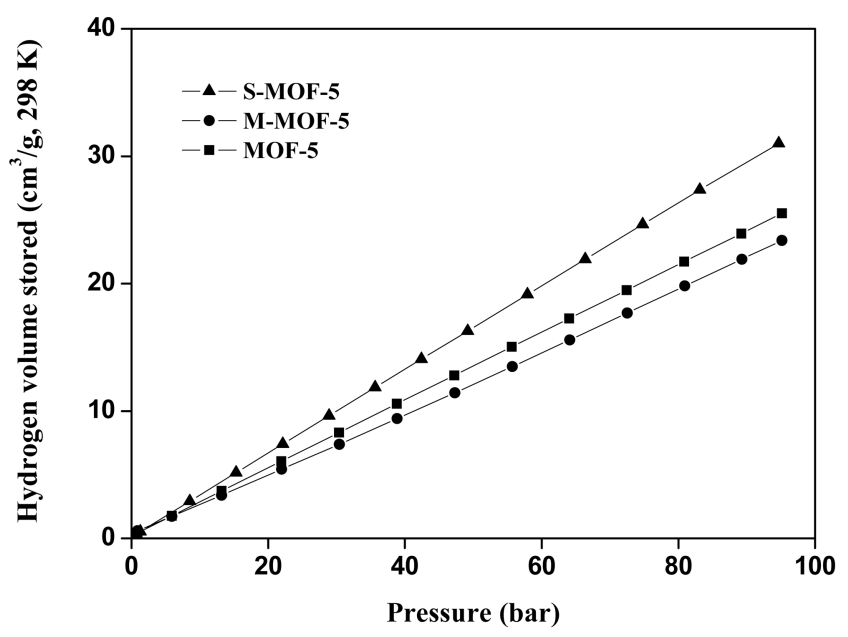

Fig. 4. Hydrogen storage capacity of MOF-5, M-MOF-5, and SMOF-5.

previously reported many researches, the results of this study correspond with those of an earlier report $[6,17,18]$, the order of hydrogen storage capacity is very similar to that of the micropore fraction, indicating that the micropore fraction plays a key role in determining hydrogen storage capacity.

This study is to investigate the possibility of improving the hydrogen storage capacity of the CNTs/MOF-5 hybrid composites, which is successfully prepared by hydrothermal synthesis with impregnation of CNTs. From the experimental results, it is observed that the presences of CNTs on MOF-5 have influence on the enhancement of hydrogen storage, resulting from the modified physicochemical properties of CNTs/MOF-5 hybrid composites. Consequently, we suggested that the CNTs/MOF-5 hybrid composites can be potentially suitable porous materials to make them as a medium for hydrogen storage.

\section{Acknowledgments}

This paper was performed for the Hydrogen Energy R\&D
Center, one of the 21st Century Frontier R\&D Program, funded by the Ministry of Education, Science and Technology of Korea.

\section{References}

[1] Schlapbach, L.; Zuttel, A. Nature 2001, 414, 353.

[2] a) Park, S.J.; Kim. B.J.; Lee, Y.S. Carbon Let. 2007, 8, 225. b) Park, S.J.; Kim. B.J.; Lee, Y.S. Carbon Let. 2007, 8, 340. c) Park, S.J.; Kim. B.J.; Lee, Y.S. Carbon Let. 2008, 9, 35.

[3] Kim, B. J.; Park, S. J. J. Colloid Interface Sci. 2007, 311, 619.

[4] Kim, B. J.; Park, S. J. J. Colloid Interface Sci. 2007, 311, 311.

[5] Leuch, L. M. Le; Bandosz, T. J. Carbon 2007, 45, 568.

[6] Kim, B. J.; Lee, Y. S.; Park, S. J. Int. J. Hydrogen Energy 2008, 33, 2254.

[7] Bénard, P.; Chahine, R. Scripta Mater. 2007, 56, 803.

[8] Rzepka, M.; Lamp, P. J. Phys. Chem. B 1998, 102, 10894.

[9] Kitagawa, S.; Kitaura, R.; Noro, S. Angew. Chem. Int. Ed. 2004, 43, 2334.

[10] Rosi, N. L.; Eckert, J.; Eddaoudi, M.; Vodak, D. T.; Kim, J.; O’Keeffe, M.; Yaghi, O. M. Science 2003, 300, 1127.

[11] Rowsell, J. L. C.; Millward, A. R.; Park, K. S.; Yaghi, O. M. J. Am. Chem. Soc. 2004, 126, 5666.

[12] Tranchemontagne, D. J.; Hunt, J. R.; Yaghi, O. M. Tetrahedron 2008, 64, 8553.

[13] Opelt, S.; Türk, S.; Dietzsch, E.; Henschel, A.; Kaskel, S.; Klemm, E. Catal. Comm. 2008, 9, 1286.

[14] Hafizovic, J.; BjØrgen, M.; Olsbye, U.; Dietzel, P.; Bordiga, S.; Prestipino, C.; Lamberti, C.; Lillerud K. P. J. Am. Chem. Soc. 2007, 129, 3612.

[15] Li, J.; Cheng S.; Zhao, Q.; Long, P.; Dong, J. Int. J. Hydrogen Energy 2009, 34, 1377.

[16] Menon, M.; Andriotis, A. N.; Froudakis, G. E. Chem. Phys. Lett. 2000, 320, 425.

[17] Brunauer, S.; Emmett, P. H.; Teller, E. J. Am. Chem. Soc. 1938, 60, 309.

[18] Kim, B. J.; Lee, Y. S.; Park, S. J. J. Colloid Interface Sci. 2008, 318, 530 . 